\title{
High Mortality Associated with Hyperglycemia, Neutrophilia, and Lymphopenia in Critically Ill Patients
}

\author{
Enrique O. Jiménez-Ibáñez, ${ }^{1,2}$ Manuel Castillejos-López, ${ }^{3}$ Andrés Hernández, ${ }^{3}$ \\ Patricia Gorocica $^{2}$ and Noé Alvarado-Vásquez ${ }^{2}$ \\ ${ }^{1}$ Graduate and Research Section, Higher School of Medicine of the National Polytechnical Institute, México City, \\ México \\ ${ }^{2}$ Department of Biochemistry, National Institute of Respiratory Diseases, "Ismael Cosío Villegas", México City, \\ México \\ ${ }^{3}$ Epidemiological Surveillance Unit, National Institute of Respiratory Diseases, "Ismael Cosío Villegas", México \\ City, México
}

A common finding in patients admitted to an Intensive Care Unit (ICU) is hyperglycemia without prior history of diabetes. This increase in blood glucose is considered a negative prognostic factor for patients in the ICU. Hence, we performed a retrospective cohort study in patients admitted at the ICU of the National Institute of Respiratory Diseases (INER) in a 7-month period; we collected data about their blood glucose concentration during their stay at the ICU. We gathered the available medical records of 30 patients out of 58 admitted to the ICU. Among the 30 patients, 21 patients survived $(70 \%)$ and 9 patients with community-acquired pneumonia (CAP) died (30\%). The 21 surviving patients included 17 patients with acute respiratory distress secondary to CAP and 4 patients with asthmatic crisis upon admission to the ICU. After admission, all patients progressed to sepsis and showed an increase in blood glucose. We detected higher glucose concentrations in deceased patients $(147 \mathrm{mg} / \mathrm{dl} \pm 4.23)$, as compared to surviving patients $(129 \mathrm{mg} / \mathrm{dl} \pm 2.17)(P<0.001)$. In addition, the percentage of lymphocytes was lower in deceased patients than that in surviving patients $(5.7$ vs. $11.8 \%, P<0.001)$, whereas percentage of neutrophils was elevated in the deceased patients $(90.7$ vs. $80.9 \%, P<0.001)$. It is therefore important to measure continuously glucose concentrations, as well as the numbers of neutrophils and lymphocytes in critically ill patients with hyperglycemia. Such a simple monitoring plan may prevent fatal complications in patients admitted to ICU.

Keywords: hyperglycemia; lymphopenia; neutrophilia; sepsis; septic shock

Tohoku J. Exp. Med., 2012, 226 (3), 213-220. _C 2012 Tohoku University Medical Press

Previous evidence shows that patients who are admitted to Intensive Care Units (ICU) have a $20 \%$ increased risk of mortality and substantial morbidity (Van den Berghe et al. 2001). This enhancement in the risk of death has been associated fundamentally with an increase in blood glucose concentration (Falciglia et al. 2009; Hirasawa et al. 2009) that is observed in patients with sepsis, as well as in critical patients with systemic diseases (Inzucchi 2006). The factors that may raise blood glucose include proinflammatory mediators such as interferon gamma (IFN- $\gamma$ ), tumor necrosis factor alpha (TNF- $\alpha$ ), and several interleukins (Inzucchi 2006; Hirasawa et al. 2009). Besides, hyperglycemia is considered a negative prognostic factor for the evolution of patients who are admitted to the ICU (Van den Berghe et al. 2001; Inzucchi 2006; Gale et al. 2007; Falciglia et al. 2009; Hirasawa et al. 2009) because of its effects on mortality, morbidity, and also by prolonging hospital stay (Van den
Berghe et al. 2001, 2006).

Current studies have tried to explain the negative effects of elevated blood glucose levels in critically ill patients (Brownle 2001). Recently, a difference in the number and activity of cells involved in the immunological response (e.g. neutrophils or monocytes) has been reported in patients with sepsis (Giamarellos-Bourboulis 2010). Additionally, increased $\mathrm{T}$ cell apoptosis associated with an impaired response to antigens for Th1 lymphocytes has been reported (Delano et al. 2011). It has been observed that in the presence of elevated glucose concentrations, polymorphonuclear and mononuclear lymphocytes exhibit an increased generation of ROS, which is linked with the oxidative injury (Mohanty et al. 2000). There is also evidence supporting a significant decrease in the total percentage of lymphocytes in patients with sepsis (Stenz and Kitabchi 2005; Venet et al. 2010), as well as impairment of

Received January 12, 2012; revision accepted for publication February 10, 2012. doi: 10.1620/tjem.226.213

Correspondence: Noé Alvarado-Vásquez, Department of Biochemistry, National Institute of Respiratory Diseases, "Ismael Cosío

Villegas". Calz. de Tlalpan 4502, Col. Sección XVI, México City 14080, Mexico, D.F.

e-mail: nnooee@gmail.com 
neutrophil degranulation associated with hyperglycemia (Stegenga et al. 2008). A high blood glucose concentration favors the activation of both neutrophils and T cells (Stentz and Kitabchi 2005; Ayilavarapu et al. 2010), and it may also induce production of superoxide by neutrophils (ShurtzSwirki et al. 2001). On the other hand, hyperglycemia observed in patients with sepsis induces functional alterations of endothelial cells (Schuetz et al. 2011), which could lead to organic dysfunction (Milman and Crandall 2001).

However, despite the aforementioned evidence, the cellular populations or a central mechanism affected in the critically ill patient by a high glucose concentration are still poorly understood. It has been suggested that oxidative stress (Stenz and Kitabchi 2005) enhances the inflammatory responses, which may affect the clotting and vascular systems during stress situations (Stegenga et al. 2008; Tsuruta et al. 2009). However, the heterogeneity of the physiological and immunological responses observed in patients with sepsis (Gustot 2011; Słowiński et al. 2011) stresses the importance of a thorough evaluation of the diverse factors involved. Thus, we performed a retrospective cohort study of patients who were admitted to the ICU at the INER from March to September 2010, to determine variations in their plasma glucose and total blood cell count that might be associated with a poor prognosis in patients admitted to the ICU.

\section{Material and Methods}

The National Institute of Respiratory Diseases (INER) "Ismael Cosío Villegas" is a tertiary and quaternary care institution in the National Health System of Mexico. The institution provides attention to patients with severe respiratory diseases. For this reason, it is important to evaluate some mechanisms involved in the clinical response of patients admitted to ICU. During the study period, 58 patients were admitted at the INER ICU. For our purposes, we obtained the complete medical records of 30 patients, but the remaining 18 were excluded because of the following reasons. Six medical records were in hospital use, eight patients did not have complete laboratory results, and the remaining four were not found at the time. Patients were divided in two groups for comparison: surviving and deceased patients. This protocol was conducted in accordance with the Helsinki Declaration and with the approval of the Ethics Committee of the INER "Ismael Cosío Villegas". All patients and control individuals signed a written informed consent.

We used a previously validated questionnaire to register information from the clinical records documenting clinical evolution, laboratory studies, and treatment received during the hospital stay. We registered age, gender, and body mass index (BMI) on admission, as well as the following laboratory variables: hemoglobin, glucose, total leucocytes, neutrophil and lymphocyte percentages, and platelet count. Total blood cell count and blood chemistry were monitored daily. It is important to mention that our ICU does not have an established standard protocol to control blood glucose. The main method used by physicians to control hyperglycemia in hospitalized patients is to administer insulin units according to the result of capillary glucose test, which does not accurately reflect central glucose concentration and is not a precise measurement.
With the obtained data, we generated an electronic database using an input mask. We used the STATA (version 11) software for statistical analysis. We applied the Shapiro Wilks and KolmogorovSmirnov test, which discarded a normal distribution of our results; for this reason, the Mann Whitney U-test was used in all cases. A posthoc analysis of the sample size using $G$ power (version 3.0.10) showed that 21 surviving and 9 deceased had enough power $(86 \%)$ to test our hypothesis.

\section{Results}

Patients admitted to the INER ICU were divided in two groups: surviving and deceased. The survived patients $(n=21)$ included 17 patients who had diagnosis of acute respiratory distress secondary to community-acquired pneumonia (CAP) and 4 patients had an initial diagnosis of asthmatic crisis upon admission to the ICU. Deceased patients $(n=9)$ had diagnosis of CAP at admission to ICU. Among the 4 patients with asthmatic crisis, two had no associated co-morbidities, one presented hyponatremia during his hospital stay, and one was a cocaine addict. In these four patients, the type of infection (viral or bacterial) was not documented. The remaining 17 surviving patients included one presented morbid obesity and H1N1 infection, one with T12 level spinal injury, one with Becker muscular dystrophy, and one with anemia. The etiological diagnosis of pneumonia was established only in two patients out of the 17 patients: one H1N1 and one Escherichia coli infection. In the group of deceased patients $(n=9)$, the cause of death was septic shock caused by pneumonia. Two patients had prior HIV infection, one of them with concomitant diabetes mellitus type II. In two patients, PCR was positive for H1N1 and in one for Influenza A. The remaining four patients had no definite etiological diagnosis although appropriate scrutiny tests had been performed.

Table 1 shows the general characteristic of the patients included in the study. There were a higher number of male patients, but gender did not show any statistical significance. $(P=0.149)$. The mean age of the surviving patients differed by roughly 10 years between both patient groups; the statistically analysis showed a slight statistical significance for this set of data $(P=0.045)$. The BMI and length of hospital stay did not show any statistical differences between both groups $(P=0.375$ and $P=0.856$, respectively).

Information obtained from clinical records revealed that all patients progressed to sepsis. Clinical features used to establish sepsis had to include at least two of the following criteria: body temperature $>38^{\circ} \mathrm{C}$ or $<36^{\circ} \mathrm{C}$, respiratory rate $>20 / \mathrm{min}$ or $\mathrm{PCO}_{2}<32 \mathrm{mmHg}$, pulse $>90 / \mathrm{min}$, leukocyte count $>12 \times 10^{3}$ or $<4 \times 10^{3}$ or over $10 \%$ of immature forms.

According to the ICU admission protocol, every patient must have a daily control of Blood Chemistry and a Total Blood Cell Count. Since we considered the elevation of blood glucose as a risk factor for the evolution of patients admitted to the ICU, we deemed it important to analyze 
Table 1. Baseline Characteristics of the Patients.

\begin{tabular}{lcc}
\hline & \multicolumn{2}{c}{ Outcome } \\
\cline { 2 - 3 } & Surviving & Deceased \\
\hline Gender & 8 & 1 \\
$\quad$ Female & 13 & 8 \\
$\quad$ Male & 21 & 9 \\
$\quad$ Total & & \\
Admission Diagnosis & $17(81 \%)$ & $9(100 \%)$ \\
CAP & $4(19 \%)$ & $0(0 \%)$ \\
Asmathic Crisis & 39 & 48 \\
Age (mean/years) & 2.559 & 3.317 \\
Std Err. & 27.99 & 26.67 \\
BMI (mean) & 1.188 & 1.143 \\
Std Err. & 21.52 & 21.89 \\
Lenght of Hospital Stay (days) & 2.920 & 5.251 \\
Std Err. & & \\
\hline
\end{tabular}

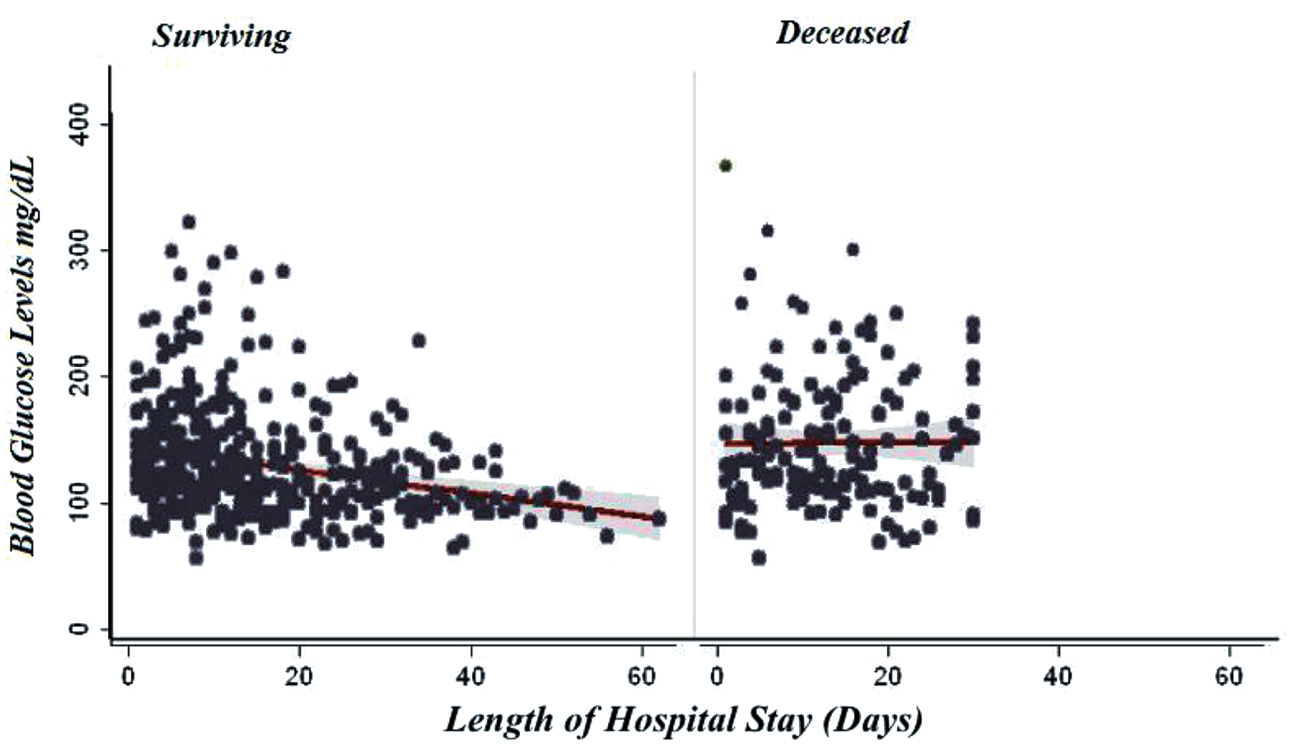

Fig. 1. Comparison of blood glucose levels between surviving and deceased patients.

The patients were divided into two groups: 21 surviving patients and 9 deceased patients. According to the ICU protocol, every patient admitted to the ICU must have everyday control of blood chemistry, including blood glucose test. Each dot represents the daily value of glucose determined in each patient. The red line represents the trend of blood glucose levels in each group. The blood glucose levels were lower in the surviving group and higher in the deceased group. The difference between the two groups is statistically significant $(P<0.001)$.

variations in blood glucose levels (Fig. 1). Blood glucose levels were higher in the group of deceased patients (mean $147 \mathrm{mg} / \mathrm{dL}, 95 \% \mathrm{CI} 138-155)$ compared to the surviving patients (mean $129 \mathrm{mg} / \mathrm{dL}, 95 \% \mathrm{CI} 125-134)$, showing statistical significance $(P<0.001)$. In Fig. 1, each dot represents the daily value of glucose determined in each patient, according to the listed group.

Hemoglobin concentration was lower in the deceased patient group (mean $10.9 \mathrm{mg} / \mathrm{dL}, 95 \%$ CI 10.6-11.2 vs. 11.8 $\mathrm{mg} / \mathrm{dL}, 95 \%$ CI 11.6-12.0 for survivors); the difference was statistically significant $(P<0.001$, Fig. 2$)$.

Our results show that the number of platelets was not significantly different between both groups of patients (mean $249 \times 10^{6}, 95 \%$ CI $238.22-261.50$, vs. $267.85 \times 10^{6}$, 95\%CI 256-279.71) ( $P=0.877$, Fig. 3). Each point in the figure represents the number of platelets determined daily in every patient.

Determination of white blood cells (WBC) revealed higher numbers in the deceased patients (mean $13.54 \times 10^{3}$, $95 \%$ CI $12.47-14-16$ ) as compared to the surviving patients 


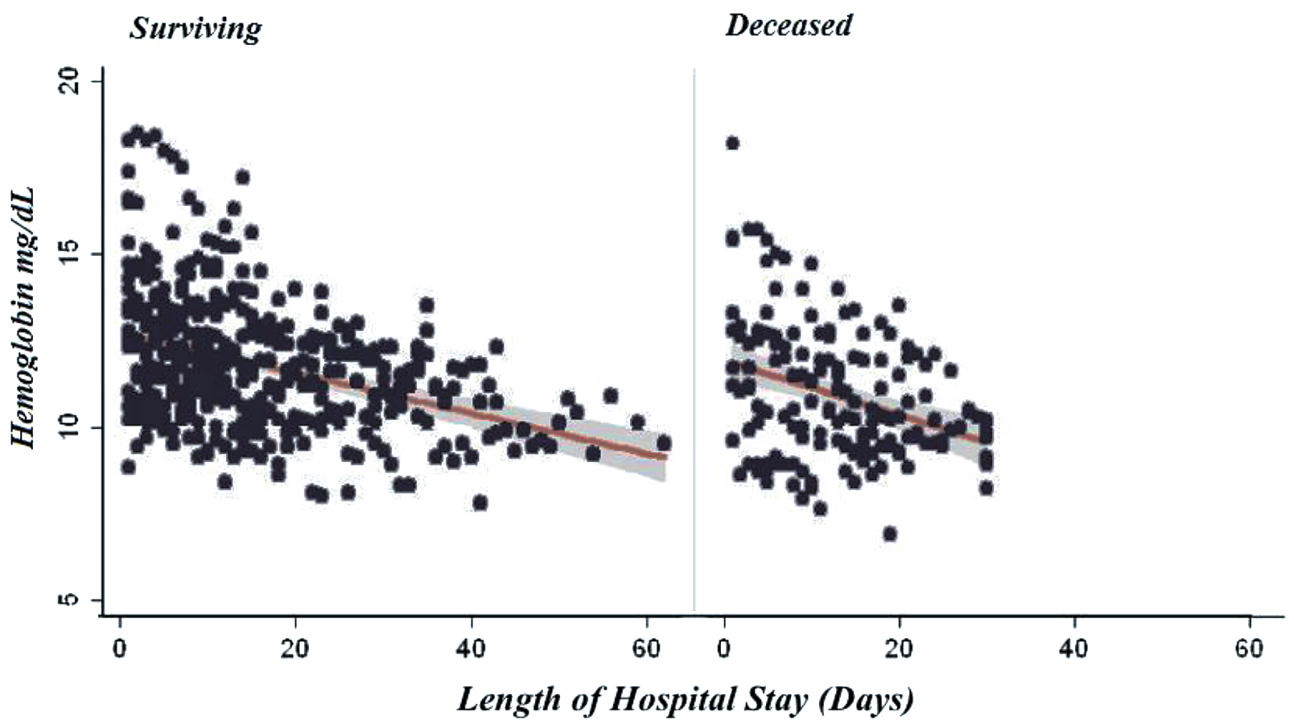

Fig. 2. Comparison of hemoglobin concentrations between surviving and deceased patients.

This graph shows the tendency of hemoglobin levels in 21 surviving patients and 9 deceased patients. Hemoglobin was measured everyday on each patient. Hemoglobin levels tended to decrease with time of hospital stay. The difference in hemoglobin concentrations is statistically significant between the two groups $(P<0.001)$.

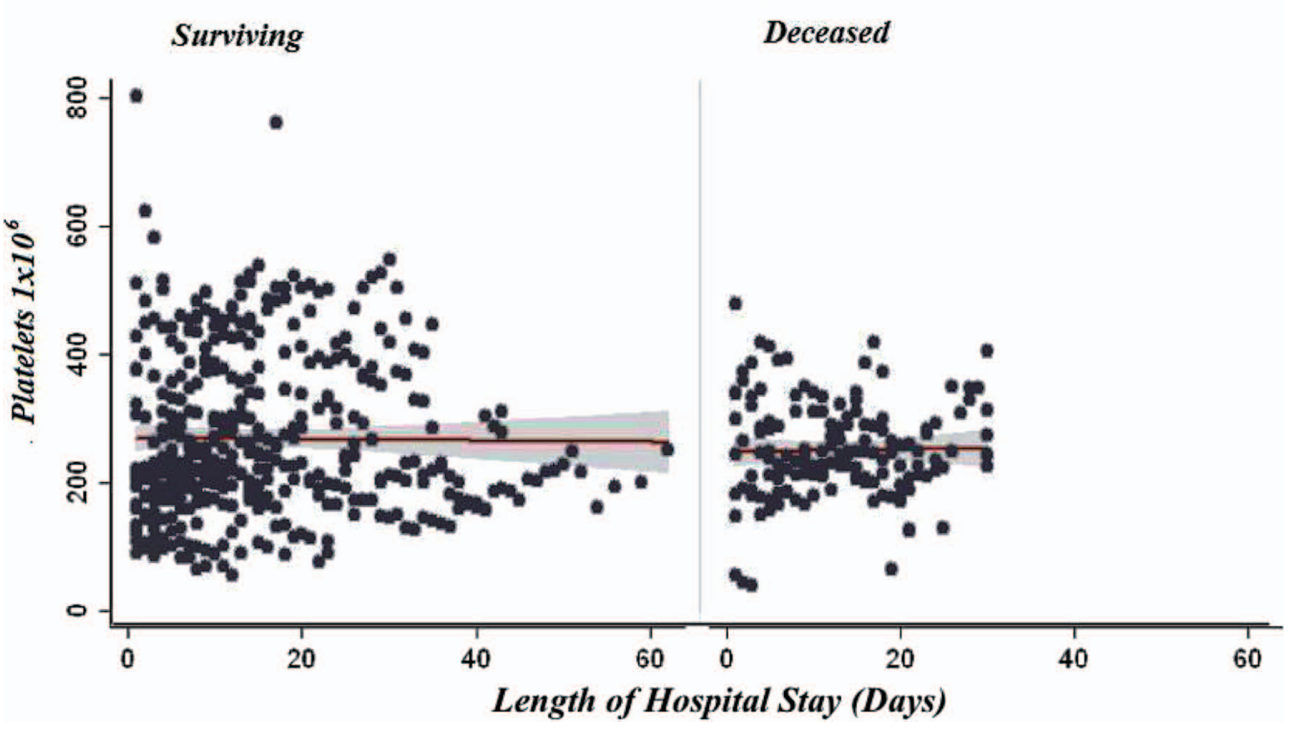

Fig. 3. Comparison of platelet numbers during hospital stay.

Each point represents the daily determination of platelets for each patient. The red line represents the trend of platelets numbers. The difference is not statistically significant between the two groups $(P=0.877)$.

(mean $10.145 \times 10^{3}, 95 \%$ CI 9.6-10.6); statistical analysis showed that the difference was significant $(P<0.001$, Fig. 4). As in previous figures, each dot represents the daily value obtained for each patient (surviving or deceased).

It is important to mention that our results showed an elevated number of neutrophils in the deceased patients, which is represented by the higher percentage of neutrophils as seen in Fig. 5 (mean 90.79\%, 95\%CI 89.8-92.1) than for the surviving group (mean $80.96 \%$, 95\%CI 79.9$81.9)$, and this difference was also statistically significant $(P$ $<0.001$, Fig. 5). Each dot represents the daily number of neutrophils determined in each patient, according to surviv- ing and deceased patients group.

Finally, we showed significant difference in lymphocytes count. Total lymphocyte percentage was significantly lower in deceased patients (mean 5.7\%, 95\%CI 4.8-6.5) as compared to surviving patients (mean $11.8 \%$, 95\%CI 11-12.5) $(P<0.001$, Fig. 6). Also, each dot in the figure represents the daily value of lymphocytes for each patient, which allowed us to evaluate the difference in the percentage of lymphocytes per group.

\section{Discussion}

Sepsis is characterized by a systemic inflammatory 


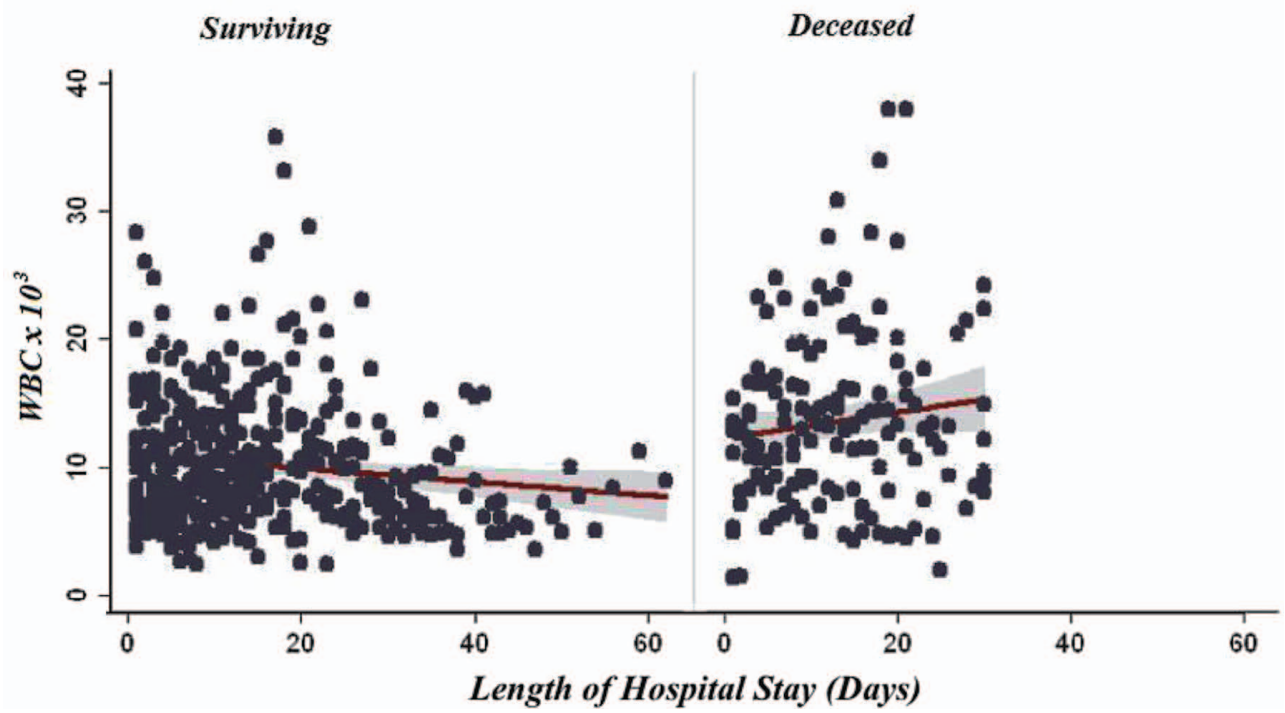

Fig. 4. Comparison of white blood cells between surviving and deceased patients.

Patients of each group underwent blood cell counting every day. Deceased patients had higher numbers of white blood cells (WBC) compared to the surviving patients $(P<0.001)$.

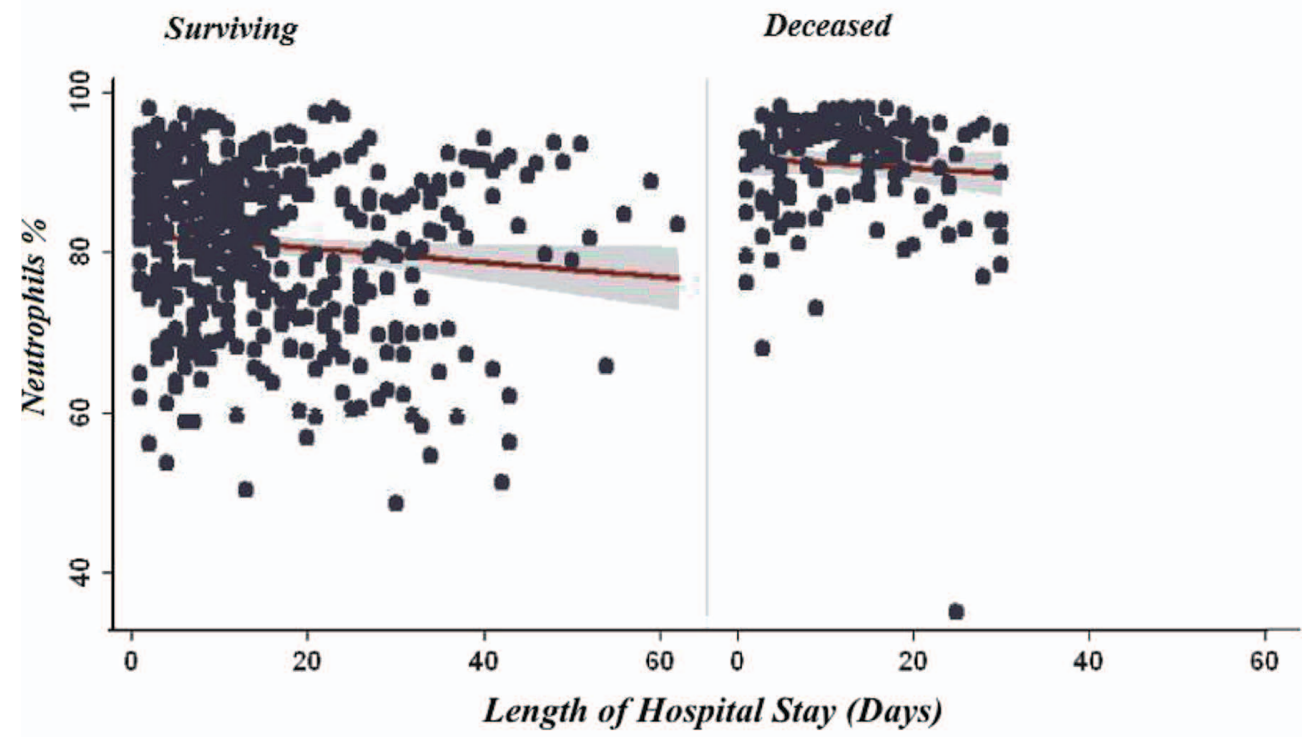

Fig. 5. Percentage of neutrophils observed in surviving and deceased patients.

Each point represents the percentage of daily neutrophils determined in each patient. The deceased patients had higher percentage of neutrophils compared to the surviving patients $(P<0.001)$.

response associated with generalized tissue injuries (Vincent et al. 2011). Evidence shows that approximately $75 \%$ of patients admitted to the ICU develop sepsis (Vincent et al. 2006). A common finding in patients admitted to the ICU is the physiological elevation of blood glucose levels (Van den Berghe et al. 2001; Inzucchi 2006; Falciglia et al. 2009; Hirasawa et al. 2009), which is considered a negative prognostic factor for clinical evolution (Gale et al. 2007). In addition, severity, as well as the duration of hyperglycemia, has been associated with worse prognosis in critically ill patients (Corstjens et al. 2006).
Our results showed higher glucose concentrations in all patients who died, with a mean of $147 \mathrm{mg} / \mathrm{dL}$. These results are consistent with previous reports showing high glucose levels as a negative prognostic factor in patients of the ICU (Van den Berghe et al. 2001, 2006). Some authors mention that high blood glucose levels at admission are predictors of poor outcomes in ICU patients (Brunkhorst et al. 2008; Kreutziger et al. 2009). Furthermore, some authors suggest that maintaining blood glucose levels between 80 and $110 \mathrm{mg} / \mathrm{dl}$ is an adequate strategy to reduce morbidity and mortality in the surgical ICU (Van den 


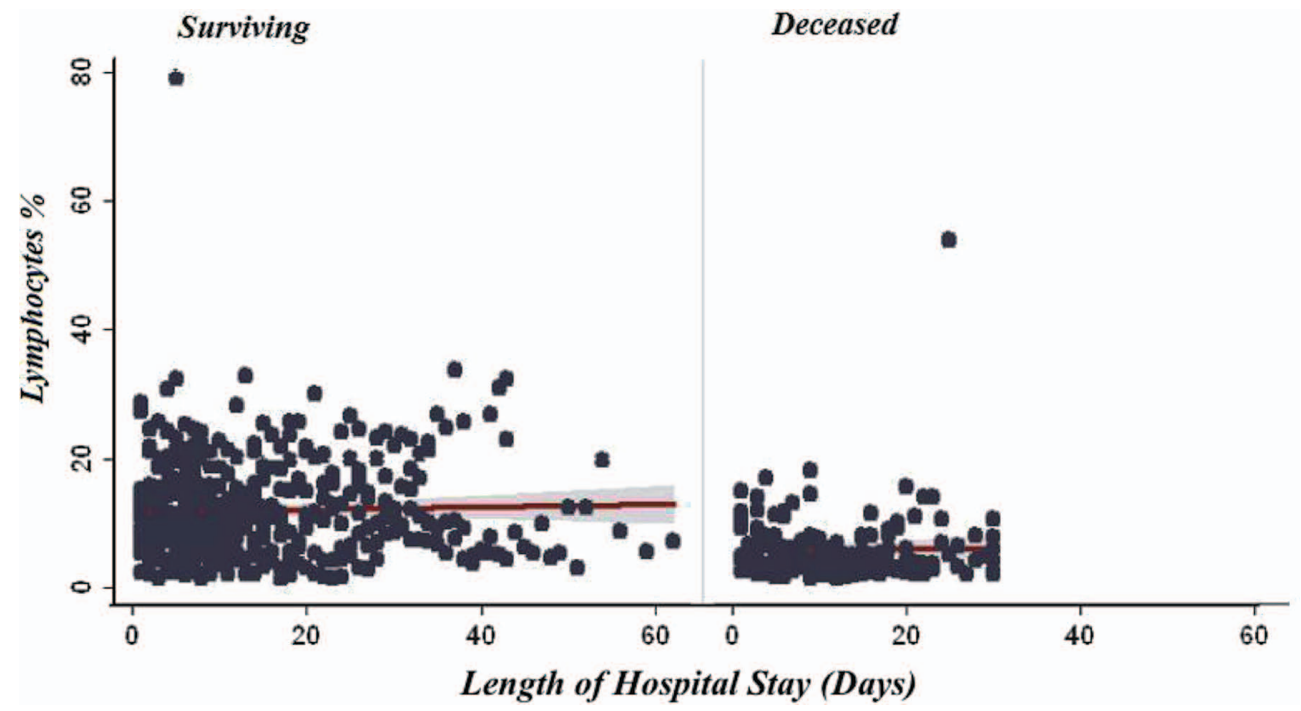

Fig. 6. Percentage of lymphocytes determined in surviving and deceased patients group.

The deceased patients showed lower lymphocyte percentage in comparison to surviving patients. Like in the other figures, each dot represents the value obtained per day from each patient. Statistical analysis shows significant differences $(P<0.001)$

Berghe et al. 2001), although, in non-surgical ICUs, these concentrations have been associated with a decrease in morbidity but not in mortality (Van den Berghe et al. 2006). Chávez-Pérez and Sánchez-Velázquez (2009) reported that a blood glucose level $<150 \mathrm{mg} / \mathrm{dl}$ was associated with the reduction in morbidity and mortality in 12 ICUs in Mexico. However, the same authors also mentioned that this parameter should be considered cautiously.

The consequences of an increase in blood glucose levels in patients with sepsis are poorly understood. Some animal models of hyperglycemia (Tsuruta et al. 2009) have shown that an elevation in blood glucose levels alone may amplify the immunological response to a given initial stimulus. In diabetic patients, it has been observed that hyperglycemia may affect the functions of neutrophils and lymphocytes (Schurtz-Swirki et al. 2001; Stenz and Kitabchi 2005). Even a mild increase in glucose levels may induce the reorganization of the lymphocyte populations (Von Känel et al. 2001) and the injury via increasing production of reactive oxygen species by lymphocytes (Stenz and Kitabchi 2005). These lines of evidence suggest that a fair control of blood glucose concentrations of ICU patients may improve their clinical evolution.

Neutrophils increased in their number in the deceased patients compared to the surviving group. We know that the function of these cells is essential in the innate immunological response to the tissue injury. Some studies have shown impaired functions of neutrophils during sepsis (Alves-Filho et al. 2006, 2008; Giamarellos-Bourboulis 2010). On the other hand, some changes in neutrophil functions have been described in healthy patients after oral administration of a glucose load (Mohanty et al. 2000). The deficient function of neutrophils is directly related to mortality in severe sepsis, clinically manifested by the progres- sion to more severe infection or lack of response to treatment. For the mentioned, the early evaluation of neutrophil activity in the patients with sepsis is an important element to consider.

Lymphocytes play a fundamental role as effector and regulatory cells in the immune response. Our results showed significant lymphopenia in the group of deceased patients. A previous report showed apoptosis as well as deficient lymphocyte activation in response to secondary stimuli in patients with sepsis (Venet et al. 2010). Our results suggest that this may happen in the patients evaluated in this study. The decrease in lymphocyte number has been associated with poor outcomes and with the increase in infectious complications in patients hospitalized in the ICU (Schroeder et al. 2001). We suggest that reduced cell number as well as their deficient function is probably linked to worse prognosis for patients in the ICU. Several lines of evidence have been published recently; they have focused on the presence of alterations of the immune response linked to the primary infectious process (Gogos et al. 2010). According to these authors, CAP could be an important factor linked with the decrease in the populations of lymphocytes. Our results show that patients with CAP and hyperglycemia worsened their prognosis. However, some authors have mentioned the presence of a blood soluble factor, which is released at an early stage and which may modulate apoptosis of CD4 lymphocytes and CD14 monocytes (Vaki et al. 2011).

Previous studies have revealed the presence of hyperglycemia, neutrophilia or lymphopenia in patients with sepsis (Alves-Filho et al. 2006; Corstjens et al. 2006; Giamarellos-Bourboulis 2010). However, the presence of these three alterations together in a same group of patients has not been reported yet. Our results showed the presence 
of all these alterations in the deceased patients admitted to the ICU of our institution, who suffered from CAP. Thus, this stresses the importance of continuously monitoring blood glucose levels, as well as the changes in percentage of lymphocytes and neutrophils, with the final objective of preventing fatal complications.

\section{Limitations of the study}

Our study has several limitations. First of all, our sample was small; the study was retrospective and included only the patients, whose complete medical records were available for a limited period of time. Secondly, we could not collect information of other variables that might have influenced the prognosis of the patients from the ICU (antibiotics, steroids, etc.). For these reasons, further studies are needed with an increased sample size. It is also reasonable to evaluate additional cell populations using surface markers to identify specific lineages and assess their function.

\section{Acknowledgments}

E.O.J.I. received graduate scholarship No. 48574 from CONACyT (National Council of Science and Technology).

\section{Conflict of Interest}

The authors have no conflict of interest.

\section{References}

Alves-Filho, J.C., de Freitas, A., Spiller, F., Souto, F.O. \& Cunha, F.Q. (2008) The role of neutrophils in severe sepsis. Shock, 30, 3-9.

Alves-Filho, J.C., Tavares-Murta, B.M., Barja-Fidalgo, C., Benjamim, C.F., Basile-Filho, A., Arraes, S.M. \& Cunha, F.Q. (2006) Neutrophil function in severe sepsis. Endocr. Metab. Immune Disord. Drug Targets, 6, 151-158.

Ayilavarapu, S., Kantarci, A., Fredman, G., Turkoglu, O., Omori, K., Liu, H., Iwata, T., Yagi, M., Hasturk, H. \& Van Dyke, T.E. (2010) Diabetes-induced oxidative stress is mediated by $\mathrm{Ca} 2+-$-independent phospholipase $\mathrm{A} 2$ in neutrophils. J. Immunol., 184, 1507-1515.

Brownle, M. (2001) Biochemistry and molecular cell biology of diabetic complications. Nature, 414, 813-820.

Brunkhorst, F.M., Engel, C., Bloos, F., Meier-Hellmann, A., Ragaller, M., Weiler, N., Moerer, O., Gruendling, M., Oppert, M., Grond, S., Olthoff, D., Jaschinski, U., John, S., Rossaint, R., Welte, T., Schaefer, M., Kern, P., Kuhnt, E., Kiehntopf, M., Hartog, C., Natanson, C., Loeffler, M. \& Reinhart, K.; German Competence Network Sepsis (SepNet). (2008) Intensive insulin therapy and Pentastarch resuscitation in severe sepsis. N. Engl. J. Med., 358, 125-139.

Chávez-Pérez, J.P. \& Sánchez-Velázquez, L.D. (2009) Glucemia como un determinante de morbimortalidad en el paciente en estado crítico. Rev. Asoc. Mex. Med. Crit. y Ter. Int., 23, 25-30.

Corstjens, A.M., van der Horst, I.C., Zijlstra, J.G., Groeneveld, A.B., Zijlstra, F., Tulleken, J.E. \& Ligtenberg, J.J. (2006) Hyperglycaemia in critically ill patients: marker or mediator of mortality? Crit. Care, 10, 216.

Delano, M.J., Thayer, T., Gabrilovich, S., Kelly-Scumpia, K.M., Winfield, R.D., Scumpia, P.O., Cuenca, A.G., Warner, E., Wallet, S.M., Wallet, M.A., O’Malley, K.A., Ramphal, R., Clare-Salzer, M., Efron, P.A., Mathews, C.E. \& Moldawer, L.L. (2011) Sepsis induces early alterations in innate immu- nity that impact mortality to secondary infection. J. Immunol., 186, 195-202.

Falciglia, M., Freyberg, R.W., Almenoff, P.L., D’Alessio, D.A. \& Render, M.L. (2009) Hyperglycemia-related mortality in critically ill patients varies with admission diagnosis. Crit. Care Med., 37, 3001-3009.

Gale, S.C., Sicoutris, C., Reilly, P.M., Schwab, C.W. \& Gracias, V.H. (2007) Poor glycemic control is associated with increased mortality in critically ill trauma patients. Ann. Surg., 73, 454-460.

Giamarellos-Bourboulis, E.J. (2010) What is the pathophysiology of the septic host upon admission? Int. J. Antimicrob. Agents, 36, S2-5.

Gogos, C., Kotsaki, A., Pelekanou, A., Giannikopoulos, G., Vaki, I. \& Maravitsa, P. (2010) Early alterations of the innate and adaptive immune statuses in sepsis according to the type of underlying infection. Crit. Care, 14, R96.

Guo, R.F. \& Ward, P.A. (2007) Role of oxidants in lung injury during sepsis. Antioxid. Redox Signal., 9, 1991-2002.

Gustot, T. (2011) Multiple organ failure in sepsis: prognosis and role of systemic inflammatory response. Curr. Opin. Crit. Care, 17, 153-159.

Hirasawa, H., Oda, S. \& Nakamura, M. (2009) Blood glucose control in patients with severe sepsis and septic shock. World J. Gastroenterol., 15, 4132-4136.

Inzucchi, S.E. (2006) Clinical practice. Management of hyperglycemia in the hospital setting. N. Engl. J. Med., 355, 19031911.

Kaufmann, I., Hoelzl, A., Schliephake, F., Hummel, T., Chouker, A., Peter, K. \& Thiel, M. (2006) Polymorphonuclear leukocyte dysfunction syndrome in patients with increasing sepsis severity. Shock, 26, 254-261.

Kreutziger, J., Wenzel, V., Kurz, A. \& Constantinescu, M.A. (2009) Admission blood glucose is an independent predictive factor for hospital mortality in polytraumatised patients. Intensive Care Med., 35, 1234-1239.

Milman, S. \& Crandall, J.P. (2001) Mechanisms of vascular complications in prediabetes. Med. Clin. North Am., 95, 309-325.

Mohanty, P., Hamouda, W., Garg, R., Aljada, A., Ghanim, H. \& Dandona, P. (2000) Glucose challenge stimulates reactive oxygen species (ROS) generation by leucocytes. J. Clin. Endocrinol. Metab., 85, 2970-2973.

Schroeder, S., Lindemann, C., Decker, D., Klaschik, S., Hering, R., Putensen, C., Hoeft, A., von Ruecker, A. \& Stuber, F. (2001) Increased Susceptibility to apoptosis in circulating lymphocytes of critically ill patients. Langenbeck's Arch. Surg., 386, 42-46.

Schuetz, P., Yano, K., Sorasaki, M., Ngo, L., St Hilaire, M., Lucas, J.M., Aird, W. \& Shapiro, N.I. (2011) Influence of diabetes on endothelial cell response during sepsis. Diabetologia, 54, 996-1003.

Shurtz-Swirski, R., Sela, S., Herskovits, A.T., Shasha, S.M., Shapiro, G., Nasser, L. \& Kristal, B. (2001) Involvement of peripheral polymorphonuclear leukocytes in oxidative stress and inflammation in type 2 diabetic patients. Diabetes Care, 24, 104-110.

Słotwiński, R., Słotwińska, S., Kędziora, S. \& Bałan, B.J. (2011) Innate Immunity Signaling Pathways: Links between Immunonutrition and Responses to Sepsis. Arch. Immunol. Ther. Exp., 59, 139-150.

Stegenga, M.E., van der Crabben, S.N., Blumer, R.M., Levi, M., Meijers, J.C., Serlie, M.J., Tanck, M.W., Sauerwein, H.P. \& van der Poll, T. (2008) Hyperglycemia enhances coagulation and reduces neutrophil degranulation, whereas hyperinsulinemia inhibits fibrinolysis during human endotoxemia. Blood, 112, 82-89.

Stentz, F.B. \& Kitabchi, A.E. (2005) Hyperglycemia-induced activation of human T-lymphocytes with de novo emer- 
gence of insulin receptors and generation of reactive oxygen species. Biochem. Biophys. Res. Commun., 335, 491-495.

Tsuruta, R., Fujita, M., Ono, T., Koda, Y., Koga, Y., Yamamoto, T., Nanba, M., Shitara, M., Kasaoka, S., Maruyama, I., Yuasa, M. \& Maekawa, T. (2009) Hyperglycemia enhances excessive superoxide anion radical generation, oxidative stress, early inflammation, and endothelial injury in forebrain ischemia/ reperfusion rats. Brain Res., 1309, 155-163.

Vaki, I., Kranidioti, H., Karagianni, V., Spyridaki, A., Kotsaki, A., Routsi, C. \& Giamarellos-Bourboulis, E.J. (2011) An early circulating factor in severe sepsis modulates apoptosis of monocytes and lymphocytes. J. Leukoc. Biol., 89, 343-349.

Van den Berghe, G., Wilmer, A., Hermans, G., Meersseman, W., Wouters, P.J., Milants, I., Van Wijngaerden, E., Bobbaers, H. \& Bouillon, R. (2006) Intensive insulin therapy in the medical ICU. N. Engl. J. Med., 354, 449-461.

Van den Berghe, G., Wouters, P., Weekers, F., Verwaest, C., Bryuninckx, F., Schetz, M., Vlasselaers, D., Ferdinande, P.,
Lauwers, P. \& Bouillon, R. (2001) Intensive insulin therapy in critically ill patients. N. Engl. J. Med., 345, 1359-1367.

Venet, F., Davin, F., Guignant, C., Larue, A., Cazalis, M.A., Darbon, R., Allombert, C., Mougin, B., Malcus, C., Poitevin-Later, F., Lepape, A. \& Monneret, G. (2010) Early assessment of leukocyte alterations at diagnosis of septic shock. Shock, 34, 358-363.

Vincent, J.L., Martinez, E.O. \& Silva, E. (2011) Evolving concepts in sepsis definitions. Crit. Care Nurs. Clin. North Am., 23, 29-39.

Vincent, J.L., Sakr, Y., Sprung, C.L., Ranieri, V.M., Reinhart, K., Gerlach, H., Moreno, R., Carlet, J., Le Gall, J.R. \& Payen, D. (2006) Sepsis in European intensive care units: results of the SOAP study. Crit. Care Med., 34, 344-353.

Von Känel, R., Mills, P.J. \& Dimsdale, J.E. (2001) Short-term hyperglycemia induces lymphopenia and lymphocyte subset redistribution. Life Sci., 69, 255-262. 